\title{
Lesões Brancas da Cavidade Oral - Uma Abordagem Estomatológica
}

\section{White Lesions of the Oral Cavity - a Stomatology Approach}

Maria Carmen Fontoura Nogueira da Cruz ${ }^{1}$, João Gabrial Farah Garcia ${ }^{2}$, Vanessa Athayde Silva Braga ${ }^{3}$, Fernanda Ferreira Lopes ${ }^{4}$, Antonio Luiz Amaral Pereira

\begin{abstract}
Introduction: White lesions of oral cavity constitute a complex group of entities, whose the main clinical characteristic is evidenced by the presence of white areas in the mouth. Your aetiology is extremely varied and some lesions don't present an only cause, but they are the result of the interaction of several factors. Aim and methods:It is intended, with this work, to accomplish a literature revision on those lesions, where it is discussed

the main white lesions of the oral cavity, your aetiology factors and clinical characteristics besides focusing necessary aspects for the establishment of the diferencial diagnosis of the referred lesions. Conclusion: It can be inferred that is necessary the knowledge of the white lesions of the oral cavity for the dentist, to established the correct diagnosis of the lesions to institute the appropriate treatment to each type, and then, the condition of the patient's health.
\end{abstract}

Keywords: Mouth, Stomatology, Etiology.

\section{Resumo}

Introdução: As lesões brancas da cavidade oral constituem um complexo conjunto de entidades, cuja principal característica clínica se evidencia pela presença de áreas esbranquiçadas na boca. Sua etiologia é extremamente variada, sendo que certas lesões não apresentam uma causa única, mas o resultado da interação de diversos fatores. Objetivo e Método: Pretende-se, com este trabalho, realizar uma revisão de literatura sobre essas lesões, onde serão discutidas as algumas das lesões brancas da cavidade oral, seus fatores etiológicos e características clínicas além de enfocar aspectos necessários para o estabelecimento do diagnóstico diferencial das referidas lesões.

Conclusão: Pode-se inferir que é necessário o conhecimento das lesões brancas da cavidade oral pelo cirurgião-dentista, a fim de que seja estabelecido o diagnóstico correto das lesões para que seja instituído o tratamento adequado a cada tipo, e então, recuperada a condição de saúde do paciente.

Palavras-chaves: Boca, Estomatologia, Etiologia
1-4-5 Prof ${ }^{\text {a }}$ Adjunto do Programa de Pós-Graduação em Odontologia da Universidade Federal do Maranhão (UFMA), doutora em Patologia Oral pela UFRN

${ }^{2-3}$ Cirurgião-dentista pela Universidade Federal do Maranhão (UFMA)

Correspondência: Maria Carmen Fontoura Nogueira da Cruz

Endereço: R. dos Rouxinóis, Condomínio Alphaville, bloco 1 apto 102 Renascença 2 - CEP 65075-630 São Luís - Ma, Brasil

E-mail: ma.carmen@uol.com.br

Data de Submissão: 16/10/2007

Data de Aceite: 04/11/2009

\section{Introdução}

As lesões brancas da mucosa oral apresentam diversas etiologias, o que leva a diagnósticos incorretos e tratamentos inapropriados. Considerando-se a sobreposição de sinais e sintomas produzidos por estas lesões, dificuldades são geradas no estabelecimento de seu diagnóstico.Torna-se necessário um exame clínico cuidadoso a fim de determinar a(s) causa(s) da lesão bem como identificar as manifestações clínicas das referidas lesões. É necessária uma detalhada investigação para determinar a(s) causa(s) da lesão, bem como a identificação de suas manifestações clínicas (NIKITAKIS, 2003; LEE; POLONOWITA, 2009).

O aspecto característico das lesões brancas da cavidade oral é derivado da dispersão da luz através de uma superfície alterada. Tais alterações podem resultar do espessamento da camada de ceratina, hiperplasia epitelial da camada de Malpighi, edema intracelular das células epiteliais e uma redução da vascularização do tecido conjuntivo subjacente. Em muitos casos, a causa destas lesões pode não estar identificada ou ser conhecida. Em outros casos, porém, pode estar evidente uma relação entre um evento ou um provável agente etiológico e a formação da lesão branca, embora o mecanismo exato não esteja compreendido ou esclarecido (REGEZI; SCIUBBA, 2000).

\section{Leucoplasia}

A Organização Mundial da Saúde (KRAMER et al., 1978) define leucoplasia como uma mancha ou placa branca da cavidade oral, não removível à raspagem, que não pode ser caracterizada clínica ou microscopicamente como outra enfermidade. Atualmente, o termo leucoplasia é utilizado apenas como um termo clínico, valendo salientar, entretanto, que este termo não traz qualquer conotação quanto às alterações histopatológicas (AXÉLL; PINDBORG; SMITH, 1996).

A leucoplasia é a lesão cancerizável mais freqüente da cavidade oral, desenvolvendo-se em qualquer região (PIMENTA et al., 2008). No entanto, a mucosa jugal, o lábio inferior e a língua têm sido as áreas mais afetadas. Sua etiologia está relacionada, na maioria dos casos, a hábitos como tabagismo, e outras vezes é considerada idiopática. Sua ocorrência se dá principalmente em pacientes de meia idade, do sexo masculino (RODRIGUES et al., 2004 ou 2000?). As lesões apresentam-se como placas que podem variar de uma área branca impalpável, fracamente translúcida, a lesões espessas, corrugadas, papilomatosas e endurecidas (GABRIEL et al., 2004). 
Do ponto de vista clínico, as leucoplasias foram divididas clinicamente em dois tipos: homogênea e não homogênea. O tipo homogênea é predominantemente branca, de superfície plana e firme, podendo exibir fendas superficiais com aspecto liso ou corrugado, de textura consistente. Já a leucoplasia não homogênea é predominantemente branca ou branco-avermelhada, que pode apresentar a superfície irregular, nodular ou exofítica (AXÉLL et al., 1996).

É sugerido um diagnóstico apurado e controle das leucoplasias dada à possibilidade de transformação maligna. É de extrema necessidade a realização de biópsia no estabelecimento do diagnóstico definitivo da lesão, considerando que seus aspectos histológicos são diversos, variando de uma hiperceratose ao carcinoma invasivo (RODRIGUES et al., 2004 ou 2000?).

O processo de diagnóstico da leucoplasia é um tanto complexo, não só pela diversidade de seu aspecto clínico, mas também pela ausência de sintomatologia, sendo geralmente descoberta em exames de rotina. Por isso, torna-se necessário fazer um diagnóstico por exclusão de outras lesões que se apresentam como placas brancas na cavidade oral (BOUQUOT, 1991).

As principais lesões a considerar-se no diagnóstico diferencial das leucoplasias são candidíase pseudomembranosa, queratose irritativa, leucoedema, líquen plano, e nevo branco esponjoso (MAO, 1997; VAN DER WALL; AXÉLL, 2002).

O primeiro passo para a realização do diagnóstico diferencial da leucoplasia na cavidade oral é determinar se a lesão pode ser removida com uma gaze. Em caso positivo, ela representará uma pseudomembrana característica da candidíase. No diagnóstico diferencial com a queimadura da mucosa, é fundamental para estabelecer o diagnóstico, relacionar a lesão branca com a obtenção de uma história exata, com a identificação de um agente químico capaz de produzir dano aos tecidos, Se existirem doença bilateral na cavidade oral, condições hereditárias, - líquen plano e lúpus eritematoso deverão ser incluídos no diagnóstico diferencial. Lesões cutâneas concomitantes falam a favor das duas últimas. Se tanto o trauma crônico quanto o uso do tabaco estiverem presentes na anamnese do paciente, a queratose irritativa ou associada ao tabaco deverão ser consideradas. A eliminação de uma causa suspeita deve resultar no esclarecimento do diagnóstico clínico (REGEZI; SCIUBBA, 2000).

\section{Queratose Irritativa}

É uma hiperplasia da camada superficial de queratina produzida por uma grande variedade de agentes que atuam sobre a mucosa de forma contínua (TOMMASI, 1989; BORAKS, 2001). Quando causada pela irritação crônica ou pelo mordiscamento da mucosa irá se apresentar clinicamente como uma placa fina e translúcida, tornando-se depois densa e branca, algumas vezes apresentando halo eritematoso que se dispõe ao longo da linha de oclusão na mucosa jugal. Embora seja uma condição benigna, é merecedora de atenção, especialmente quando o seu aspecto clínico é verrucoso, considerando que pode apresentar histologicamente características de uma displasia epitelial. Mesmo com o diagnóstico clínico e suspensão do hábito pode levar até alguns anos para desaparecer completamente (CARVALHO et al., 2000).

A anamnese cuidadosa e o exame fisico devem indicar a natureza da lesão. Se o profissional tiver certeza de uma causa traumática, a biópsia não será necessária. $O$ paciente deve ser aconselhado a abolir o hábito causador. Com o tempo, a lesão deve desaparecer ou, pelo menos, reduzir em intensidade, confirmando o diagnóstico. A resolução da lesão também pode permitir a revelação de qualquer lesão subjacente que pode não estar relacionada a traumatismo (REGEZI; SCIUBBA, 2000).

\section{Líquen Plano}

O líquen plano é uma doença mucocutânea inflamatória crônica comum, que acomete a pele, a mucosa ou ambas e que apresenta manifestações orais extremamente variáveis. Apresentações orais são mais freqüentes do que as cutâneas e tendem a ser mais persistentes e mais resistentes ao tratamento. Tais apresentações orais podem representar os primeiros sinais da doença, bem como surgirem apenas após as aparições em pele. Pacientes com líquen plano oral freqüentemente têm concomitantemente a doença em um ou mais sítios extra-orais, fato que releva a importância de um acompanhamento multidisciplinar (MOLLAOGLU, 2000; LOZADA, 2000). As mulheres são mais afetadas do que os homens com uma taxa aproximada de 2:1, principalmente na idade adulta e em pacientes mais idosos (EISEN 2002).

A etiopatogenia do líquen plano oral ainda é desconhecida, embora seja levantada a hipótese de mecanismos imunológicos, onde auto-antígenos podem ser reconhecidos como estranhos, resultando em uma resposta auto-imune. A destruição da camada de células basais do epitélio também pode ser explicada pela presença de antígenos reconhecidos erroneamente como linfócitos $\mathrm{T}$ do hospedeito que podem se tornar células citotóxicas para as células epiteliais em uma reação hiperimune (CARBONE et al, 2009). Entretanto, é citada a possibilidade de transformação maligna durante sua evolução (FEMOPASE et al., 1997). Torna-se necessária proservação criteriosa de todos os pacientes com líquen plano oral por um longo período de tempo (AMORIM et al., 2002)..

Apresenta-se tipicamente sob a forma de lesões bilaterais brancas (placas ou áreas reticulares), especialmente na mucosa jugal e na língua, podendo também afetar o rebordo alveolar, a gengiva e o palato. Estas lesões brancas são geralmente assintomáticas (AZUL; TRANCOSO, 2006).

A forma de aparecimento mais comum das lesões no líquen plano oral é a reticular que aparece como estrias brancas finas com aspecto rendilhado, conhecidas como estrias de Wickham, sendo normalmente este tipo assintomático e freqüentemente descoberto incidentalmente durante um exame oral de rotina. Já as formas atrófica, erosiva e bolhosa usualmente são associadas à sensação de ardência e em alguns casos podem causar dor severa nos pacientes. O líquen plano oral é uma doença de natureza crônica que persiste por longos períodos em alguns pacientes e poucos sofrem remissão completa (MOLLAOGLU, 2000).

O diagnóstico é geralmente clínico, mas o exame anátomo-patológico é importante para excluir, entre outras situações, leucoplasia, lúpus eritematoso sistêmico e doenças bolhosas (AZUL; TRANCOSO, 2006).

\section{Candidíase}

É a infecção micótica mais comum da cavidade oral causada pelo fungo do gênero Candida, especialmente $C$. albicans embora outras espécies possam estar envolvidas (SCHERMA et al., 2004; RICHARDSON; RAUTEMAA, 2009). A C. albicans é considerada o principal agente etiológico da candidíase, infecção oportunista relacionada a fatores locais e sistêmicos (ARENDORF WALKER, 1979; CANNON, 1995).

Quando ocorre ruptura do equilíbrio biológico, geralmente resultante de fatores predisponentes (patológicos, fisiológicos, imunológicos e mecânicos), há um aumento na multiplicação e/ou invasão de leveduras nos tecidos, instalando-se então a infecção. Geralmente está associada a indivíduos debilitados, imunocomprometidos ou que estejam fazendo uso abusivo de antibióticos, trazendo assim conseqüências mais graves (GHANNOUM; ABU-ELTEEN, 1990).

Clinicamente se apresenta sob as formas: aguda (pseudomembranosa, atrófica), crônica (atrófica, hiperplásica), 
muco-cutânea (localizada, familial, associada à síndromes). Caracteristicamente, as lesões bucais da candidíase aguda são placas ou nódulos brancos, entre moles e gelatinosos, que crescem centrifugamente e confluem. As placas são compostas por fungos, resíduos ceratóticos, células inflamatórias, células epiteliais descamadas, bactérias e fibrina. A remoção das placas ou pseudomembranas com uma compressa de gaze deixa uma superfície eritematosa, erosada ou ulcerada, frequentemente visível. As infecções pela Cândida devem ser diferenciadas de várias entidades, incluindo a escara associada com queimaduras químicas, as ulcerações traumáticas, as placas mucosas da sífilis e as lesões ceratóticas. Quando estão presentes lesões vermelhas isoladas da candidíase atrófica aguda, elas devem ser diferenciadas das reações medicamentosas e das queimaduras térmicas (REGEZI; SCIUBBA, 2000).

O seu diagnóstico é eminentemente clínico, embora eventualmente pode-se fazer uso de culturas (CARVALHO et al., 2000).

É uma situação freqüente que se caracteriza pelo desaparecimento das papilas filiformes com a manutenção das papilas fungiformes, tanto do dorso quanto das bordas laterais da língua. Clinicamente, observam-se áreas vermelhas (semelhantes a mapas) por vezes, rodeadas por zonas elevadas esbranquiçadas de contorno bem definido. As áreas despapiladas persistem desta forma por um período variável, normalizam espontaneamente e aparecem em outro lugar, configurando o aspecto migratório da lesão. O diagnóstico é clínico e as lesões são patognomônicas. Geralmente assintomática, esta lesão pode provocar incômodo ou ardor nas zonas despapiladas, sobretudo com a ingestão de alimentos ácidos ou picantes. É importante não confundir esta situação com uma candidíase da língua, pois muitas vezes, os pacientes são submetidos a tratamentos prolongados com antifúngicos, desnecessariamente (FEMIANO, 2001; AZUL; TRANCOSO, 2006).

A etiologia da língua geográfica ainda permanece desconhecida, entretanto é referida com maior freqüência em pacientes com estresse psicológico e aparece com um aumento aproximado de $10 \%$ nas pessoas com psoríase cutânea (KRAETHER NETO et al., 2004).

\section{Leucoedema}

É um achado clínico comum, encontrado em cerca de 3/4 da população negra, sendo usualmente um achado acidental (TOMMASI, 1989). Ainda não foi estabelecida a origem do leucoedema, parecendo ser uma anomalia de desenvolvimento, mas pode estar relacionada com má higiene bucal e padrões anormais de mastigação. É assintomático, de distribuição simétrica e encontrado na mucosa jugal, como uma área esbranquiçada ou leitosa. Nos casos mais acentuados, pode-se notar uma alteração da textura da superfície. Um critério clínico extremamente importante no diagnóstico do leucoedema é o fato de que ao se distender a mucosa jugal, a lesão desaparece, retornando quando deixamos de exercer a tração (CERRI; SILVA, 2005).

\section{Considerações Finais}

Diante do exposto, vale ressaltar que as lesões brancas da cavidade oral constituem um grupo heterogêneo de processos caracterizados clinicamente por sua coloração esbranquiçada, de etiologia variada, particular para cada tipo de lesão. Ratifica-se, com base na literatura pertinente, que certas lesões não apresentam uma causa isolada, mas que resultam da interação de diversos fatores. Algumas são de origem genética, outras são resultantes da ação irritativa de agentes mecânicos, físicos e químicos, outras microbianas. Certos quadros parecem ser de origem psicogênica e, finalmente, um certo número é de etiologia duvidosa ou totalmente desconhecida. Ressalta-se, ainda que, estas lesões são cada vez mais freqüentes na clínica odontológica e apresentam um vasto espectro de manifestações clínicas. Dessa forma, são, por vezes, de difícil diagnóstico, e então, tratadas de maneira incorreta. Neste contexto, o levantamento da história tem importância considerável no estabelecimento do diagnóstico das lesões brancas da mucosa oral, bem como as informações clínicas para que se faça o diagnóstico diferencial entre as mesmas, considerando-se duração do processo, ocorrência familiar, identificação de hábitos, lesões cutâneas associadas, ação de agentes físico, químicos ou biológicos bem como reação psicogênica. Considerando-se que, em determinadas situações, as lesões orais podem apresentar aspectos clínicos muito semelhantes, o cirurgião-dentista deverá lançar mão de características próprias a cada tipo de lesão para seu diagnóstico diferencial (Tabela 1).

Tabela 1. Diagnóstico diferencial das lesões brancas da cavidade oral

\begin{tabular}{|c|c|c|c|}
\hline Lesão & Aspecto clínico & Etiologia & Curso clínico \\
\hline Leucoplasia & $\begin{array}{c}\text { Placa branca assintomática que não cede à } \\
\text { raspagem, de aspecto corrugado, fissurado, } \\
\text { pilomatoso e endurecido. Os homens são } \\
\text { mais afetados }\end{array}$ & $\begin{array}{c}\text { Sua etiologia está relacionada, na } \\
\text { maioria dos casos, a hábitos como } \\
\text { tabagismo, e outras vezes é consi- } \\
\text { derada idiopática }\end{array}$ & $\begin{array}{l}\text { A recorrência, após algum } \\
\text { tipo de remoção ci-rúrgica, } \\
\text { não é rara }\end{array}$ \\
\hline $\begin{array}{l}\text { Queratose } \\
\text { irritativa }\end{array}$ & Placa densa e branca. Condição benigna & Irritativa & $\begin{array}{c}\text { Pode regredir, se a causa for } \\
\text { eliminada }\end{array}$ \\
\hline Líquen Plano & $\begin{array}{c}\text { Doença mucocutânea. Estrias brancas } \\
\text { bilaterais (de Wickham); assinto-mático, } \\
\text { exceto quando há erosões. A mucosa jugal é } \\
\text { mais afetada }\end{array}$ & $\begin{array}{c}\text { Desconhecida, embora seja levantada a } \\
\text { hipótese de ser uma condição auto- } \\
\text { imune }\end{array}$ & $\begin{array}{l}\text { Pode regredir após alguns } \\
\text { anos }\end{array}$ \\
\hline Candidíase & $\begin{array}{c}\text { Infecção micótica. Placas ou nódulos brancos, } \\
\text { entre moles e gelatinosos, deixam uma } \\
\text { superfície eritematosa, erosada ou ulcerada } \\
\text { quando removida. }\end{array}$ & Fungo do gênero Candida & $\begin{array}{c}\text { Geralmente de-saparece em } \\
1 \text { ou } 2 \text { semanas após o } \\
\text { tratamen-to }\end{array}$ \\
\hline $\begin{array}{l}\text { Língua } \\
\text { geográfica }\end{array}$ & $\begin{array}{l}\text { Lesões brancas, anulares, com centros } \\
\text { vermelhos atróficos. É comum e às vezes é } \\
\text { dolorosa }\end{array}$ & Desconhecida & $\begin{array}{l}\text { Pode desaparecer } \\
\text { espontaneamente }\end{array}$ \\
\hline Leucoedema & $\begin{array}{l}\text { Achado comum na população negra, } \\
\text { opacificação uniforme bilateral da mucosa } \\
\text { jugal, de natureza assintomática }\end{array}$ & Desconhecida & $\begin{array}{l}\text { Não possui signi-ficado } \\
\text { clínico }\end{array}$ \\
\hline
\end{tabular}


Pode-se inferir que é necessário o conhecimento das lesões brancas da cavidade oral pelo profissional da Odontologia, a fim de que seja estabelecido o diagnóstico correto das lesões para que seja instituído o tratamento adequado a cada tipo, e então, restaurada a condição de saúde do paciente.

\section{Referências}

AMORIM, R.F.B. et al. Líquen Plano Oral em Paciente Diabética Associado a Carcinoma Mucoepidermóide. Rev. Paul. Odontol., São Paulo, v. 24, n. 4, p. 8-14, jul./ago. 2002.

ARENDORF, T.M.; WALKER, D.M. Oral Candidal Populations in Health and Disease. Br. Dent. J., London, v. 147, no. 10, p. $267-$ 272, Nov.1979.

AXÉLL, T. et al. Oral White Lesions with Special Reference to Precancerous and Tobacco-related Lesions: Conclusions of an International Symposium Held in Uppsala, Sweden, May 18-21, 1994. J. Oral Pathol. Med., Copenhagen, v. 25, no. 2, p. 49-54, Feb. 1996

AZUL, A. M.; TRANCOSO, P. F. Patologia mais Frequente da Mucosa Oral. Rev. Port. Clin. Geral, [S.I.], v. 2, p. 369-377, maio/jun. 2006.

BORAKS, S. Diagnóstico Bucal. 3.ed. São Paulo: Artes Médicas, 2001.

BOUQUOT, M. Reviewing oral Leukoplakia: Clinical Concepts for the 1990`s. J. Am. Dent. Assoc., Chicago, v. 122, no. 7, p. 80-82, June 1991.

CANNON, R.D. et al. Oral Candida: Clearance, Colonization, or Candidiasis? J. Dent. Res., Chicago, v. 74, no. 5, p. 1152- 1161, May 1995.

CARBONE, M. et al. Course of Oral Lichen Planus: a Retrospective Study of 808 Northern Italian Patients. Oral Dis., Munksgaard, v. 15, no.3, p. 235-243, Apr. 2009

CARVALHO, A. T. et al. Estomatologia: Atualização para o Clínico Geral. Parte II: Lesões Brancas da Cavidade Oral. An. Fac. Odontol. UFPE, Recife, v. 10, n. 2, p. 179-186, 2000.

CERRI, A.; SILVA, C. E. X. dos S. R. da. Desvios de Normalidade da Cavidade Bucal. 2005. Disponível em: <http://www.guiaodonto.com.br/ver_artigo.asp?codigo=122>. Acesso em: 31 jul. 2007.

EISEN, D. The Clinical Features, Malignant Potencial, and Systemic Associations of Oral Lichen Planus: a Study of 723 Patients. J. Am. Acad. Dermatol., St. Louis, v. 46, no. 2, p. 207-214, Feb. 2002.

FEMIANO, F. Geographic Tongue (Migrant Glossitis) and Psoriasis. Minerva Stomatol., Torino, v. 50, no. 6, p. 213-217, June 2001.

FEMOPASE, F. L. et al. A Comparative Study of Oral Lichen Planus and Leukoplakia in Two Argentine Populations. Acta Odontol. Latinoam., Buenos Aires, v. 10, n. 2, p. 89-99, 1997.

GABRIEL, J. G. et al. Considerações Gerais e Abordagem Clínica da Leucoplasia Oral. Rev. Bras. Patol. Oral, Natal, v. 3, n. 4, p. 187-194, out./dez. 2004.

GHANNOUM, M. A.; ABU-ELTEEN, K. H. Pathogenicity Determinants of Candida. Mycoses, Berlin, v. 33, no. 6, p. 265-282, June 1990.
LEE, K.H.; POLONOWITA, A.D. Oral White Lesions: Pitfalls of Diagnosis. Med. J. Aust., Sidney, v.190, n. 5, p. 274-277, Mar. 2009.

LOZADA, N. F. Oral Lichen Planus and Oral Câncer: is there Enough Epidemiologic Evidence? Oral Surg. Oral Med. Oral Pathol. Oral Radiol. Endod., St. Louis, v. 89, no. 3, p. 265-266, Mar. 2000.

MAO, L. Leukoplakia: Molecular Understanding of Pre-malignant Lesion and Implications for Clinical Management. Mol. Med. Today, Cambridge, v. 3, no. 10, p. 442-448, Oct. 1997.

MOLLAOGLU, N. Oral Lichen Planus: a Review. Br. J. Oral Maxillofac. Surg., Edinburgh, v. 38, no. 4, p. 370-377, Aug. 2000.

KRAETHER NETO, L. et al. Relação entre Língua Geográfica e Psoríase. Rev. Bras. Patol. Oral, Natal, v. 3, n. 1, p. 32-35, jan./mar. 2004.

KRAMER, I.R.H. et al. Definition of Leukoplakia and Related Llesions: an Aid to Studies on Oral Precancer. Oral Surg. Oral Med. Oral Pathol., St. Louis, v. 46, no. 4, p. 518-539, Oct. 1978.

NEVILLE, B. D. et al. Patologia Oral \& Maxilofacial. Rio de Janeiro: Guanabara Koogan, 1998.

NIKITAKIS, N. Oral Soft Tissue Lesions: A Guide to Differential Diagnosis. Part I: Introduction and Changes in Color. Braz. J. Oral Sci., Piracicaba, v. 2, no. 6, p. 291-299, July/Sept. 2003.

PIMENTA, F.J. et al. Molecular Alterations in the Tumor Suppressor Gene WWOX in Oral Leukoplakias. Oral Oncol., Oxford, v. 44, no. 8, p. $753-8$, Aug. 2008

REGEZI, J.A.; SCIUBBA, J. J. Patologia Bucal: Correlações Clinicopatológicas. 3. ed. Rio de Janeiro: Guanabara Koogan, 2000.

RICHARDSON, M.; RAUTEMAA, R. How the Host Fights against Candida Infections. Front Biosci, Tampa, v.14, p. 4363-75, June 2009.

RODRIGUES, T. L. C. et al. Leucoplasias Bucais: Relação Clínicohistopatológica. Pesqui. Odontol. Bras., São Paulo, v. 14, n. 4, p. 357-361, out./dez. 2000.

SCHERMA, A. P. et al. Presença de Candida spp. na Cavidade Bucal de Lactentes Durante os Primeiros Quatro Meses de Vida. Cienc. Odontol. Bras., São Paulo, v. 7, n. 3, p. 79-86, jul./dez. 2004.

TOMMASI, A. F. Diagnóstico em Patologia Bucal. 2. ed. São Paulo: Pancast, 1989.

VAN DER WALL, I.; AXÉLL, T. Oral Leukoplakia: a Proposal for Uniform Reporting. Oral Oncol, Oxford, v. 38, no. 6, p. 521-526, Sept. 2002. 